Ultrasonic classification of thin layers within multi-layered materials

This article has been downloaded from IOPscience. Please scroll down to see the full text article.

2010 Meas. Sci. Technol. 21015701

(http://iopscience.iop.org/0957-0233/21/1/015701)

The Table of Contents and more related content is available

Download details:

IP Address: 130.240.45.104

The article was downloaded on 09/04/2010 at 06:46

Please note that terms and conditions apply. 


\title{
Ultrasonic classification of thin layers within multi-layered materials
}

\author{
F Hägglund ${ }^{1}$, J E Carlson ${ }^{2}$ and T Andersson ${ }^{2}$ \\ ${ }^{1}$ EISLAB, Department of Computer Science and Electrical Engineering, Luleå University of \\ Technology, SE-971 87 Luleå, Sweden \\ ${ }^{2}$ Division of Systems and Interaction, Department of Computer Science and Electrical Engineering, \\ Luleå University of Technology, SE-971 87 Luleå, Sweden \\ E-mail: Fredrik.Hagglund@ltu.se
}

Received 20 July 2009, in final form 15 October 2009

Published 16 November 2009

Online at stacks.iop.org/MST/21/015701

\begin{abstract}
Methods for non-destructive inspection of layered materials are becoming more and more popular as a way of assuring product integrity and quality. In this paper, we present a model-based technique using ultrasonic measurements for classification of thin bonding layers within three-layered materials. This could be, for example, an adhesive bond between two thin plates, where the integrity of the bonding layer needs to be evaluated. The method is based on a model of the wave propagation of pulse-echo ultrasound that first reduces the measured data to a few parameters for each measured point. The model parameters are then fed into a statistical classifier that assigns the bonding layer to one of a set of predefined classes. In this paper, two glass plates are bonded together with construction silicone, and the classifiers are trained to determine if the bonding layer is intact or if it contains regions of air or water. Two different classification methods are evaluated: nominal logistic regression and discriminant analysis. The former is slightly more computationally demanding but, as the results show, it performs better when the model parameters cannot be assumed to belong to a multivariate Gaussian distribution. The performance of the classifiers is evaluated using both simulations and real measurements.
\end{abstract}

Keywords: multi-layered materials, ultrasonic measurements, parameter estimation, statistical classification

\section{Introduction}

Non-destructive testing of materials using ultrasound has a wide range of applications in the area of process control; see for example [1-3]. In this paper, we look at the specific case of materials consisting of three layers with a thin middle layer. The task is to estimate a set of properties of each layer and classify the material samples based on some or all of these.

The measurement technique is based on pulse-echo ultrasound where the reflected echoes from within the material structure are used to extract some features of the layers. These features then serve as input to a classifier, which assigns each measured point to one of the predefined classes. As will be shown, this method enables us to detect and discriminate between a number of different flaws in the material.

However, thin multi-layered materials will give rise to reverberant and overlapping ultrasound waveforms, making the characterization difficult. Several different methods to handle multi-layered structures have been proposed [4-10]. In this study, we use a parametric model of the wave propagation to analyze the multi-layered material. The parameters of the model are directly connected to physical properties of the investigated material [11]. The subsequent analysis and classification are then performed on the model parameters rather than on the entire waveform, which enables cost-effective storage and fast processing, necessary for applications in process control.

We will evaluate two different classification methods in this paper: nominal logistic regression and discriminant analysis [12]. Both methods use a single nominal categorydependent variable, meaning that the output of the classifier is a single number, representing one of the categories. The input to the classifiers, called the explanatory variables, are the parameters of the wave propagation model, estimated 


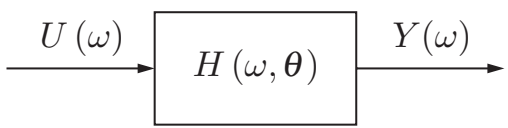

Figure 1. Linear systems representation of the wave propagation model.

from the measured ultrasonic waveforms. Logistic regression is known to perform better when the explanatory variables cannot be assumed to belong to a multivariate Gaussian distribution. Discriminant analysis, on the other hand, is slightly less computationally demanding, but in general the analysis requires the assumption of normally distributed explanatory variables to be valid. Since this cannot be guaranteed, both methods are evaluated.

The classification methods proposed in this paper are verified using both simulations and measurements. The purpose of the simulations is to investigate the limitations of the layer thickness classification. The measurements are used to evaluate the classifier performance on typical materials.

The remainder of the paper is organized as follows. In section 2.1, a summary of the multi-layered model is given, together with a description of the parametrization and the parameter estimation procedure. The classification algorithms are described in section 2.2, and a description of the experimental set-up is given in section 3 . The results are divided into simulation results in section 4 and measurement results in section 5. Finally, in sections 6 and 7 the discussion and conclusions are presented.

\section{Theory}

This section begins with a brief summary of the parametric model described in previous studies [11]. The classifiers are then outlined, followed by a discussion on how to select which features are to be used by the classifiers.

\subsection{Parametric wave-propagation model}

Following the steps in [11], the received ultrasound signal from a point on the surface of a three-layered material can be modeled as a linear system. Given a reference echo and the parameters of the model, it has been shown that the received waveform can be accurately reconstructed, suggesting that all significant mechanisms of the wave propagation are captured by the model.

So, assuming that linear acoustics apply, the received echo can be modeled as the output of the linear system in figure 1, as

$$
Y(\omega)=H(\omega, \boldsymbol{\theta}) U(\omega),
$$

where $U(\omega)$ is the discrete Fourier transform of the input signal (e.g. the transmitted ultrasound pulse) and where

$$
\begin{aligned}
& H(\omega, \boldsymbol{\theta})=R_{43} M_{4}^{2}+\left(1-R_{43}^{2}\right) M_{4}^{2} M_{3}^{2} \\
& \quad \times\left(R_{32}+R_{21} M_{2}^{2}+R_{32} R_{21} R_{10} M_{1}^{2}+R_{10} M_{2}^{2} M_{1}^{2}\right) \\
& \quad \times\left(1+R_{43} R_{32} M_{3}^{2}+R_{32} R_{21} M_{2}^{2}+R_{21} R_{10} M_{1}^{2}\right. \\
& \quad+R_{43} R_{32} R_{21} R_{10} M_{3}^{2} M_{1}^{2}+R_{32} R_{10} M_{2}^{2} M_{1}^{2} \\
& \left.\quad+R_{43} R_{21} M_{3}^{2} M_{2}^{2}+R_{43} R_{10} M_{3}^{2} M_{2}^{2} M_{1}^{2}\right)^{-1}
\end{aligned}
$$

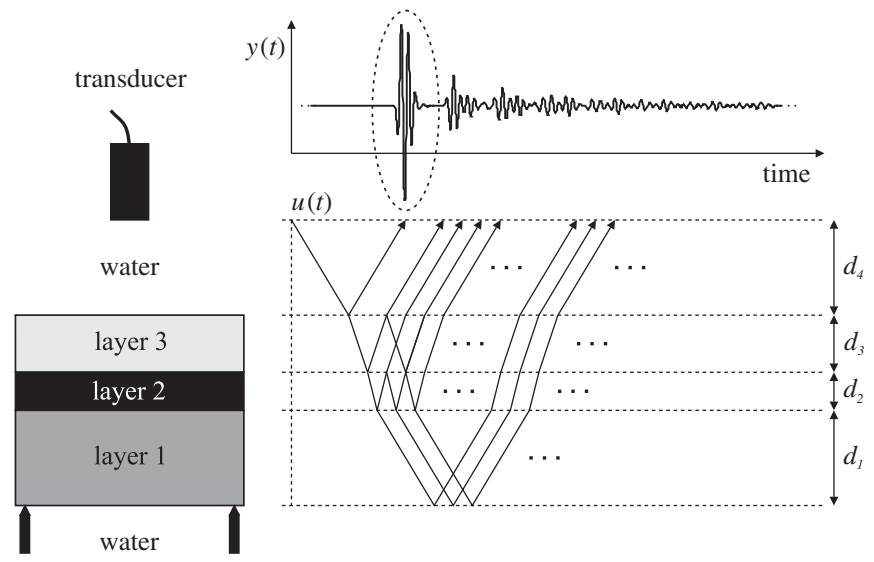

Figure 2. The experimental set-up in a pulse-echo configuration. The waveforms and the notations are in the time domain, where $u(t)$ represents the input signal and $y(t)$ is the entire output signal (summation of all reflected echoes). The DFT of the entire waveform $y(t)$ is used as the output signal, $Y(\omega)$, and the DFT of the first echo (circled with a dashed line) is used as the input signal, $U(\omega)$. The layer thicknesses in this figure are only descriptive and not the actual thicknesses of the layers in the material.

is the transfer function, where the collected exponential terms are modeled as

$$
M_{q}=\mathrm{e}^{-\mathrm{j} \omega \tau_{q}-\alpha_{q} \omega^{2} d_{q}},
$$

where $d_{q}$ is the thickness of layer $q$. The properties in the water buffer region $M_{4}$ are assumed to be known (tabulated values) [13]. The parameter vector is then $\boldsymbol{\theta}=$

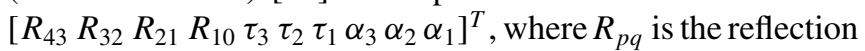
coefficient at the boundary between layers $p$ and $q, \tau_{q}$ is the time-of-flight and $\alpha_{q}$ is the attenuation coefficient inside layer $q$. In this case, the echo from the top surface of the material (see figure 2) is used as the input, after compensating for the time-of-flight and the attenuation in the water buffer region. A complete description of the details of the model can be found in [11]. Note that, in the figures, the time-domain representations are plotted, although all modeling is done in the frequency domain. A description of how the measurements over the surface of the object were conducted is given in sections 3 and 5.1 .

The maximum likelihood estimate, $\widehat{\boldsymbol{\theta}}$, of the parameter vector $\boldsymbol{\theta}$ is then obtained using the algorithm presented in [11].

\subsection{Classification}

In this section, the principles of categorical classification rules are discussed and the two classification methods are briefly explained for multi-categorical classification. A short discussion on the method for selecting which features are to be used by the classification rules is also given.

2.2.1. Categorical classification rules. In statistical categorical classification, the goal is to determine which category (subgroup) an item (measurement, material, etc) belongs to, using the item's explanatory variables (features, properties, model parameters, etc). The number and the type of 
categories are predefined, and the items belonging to different categories should have different properties. In this study, the items are the ultrasonic measurement points on an object, and the explanatory variables are the estimated model parameters for each measurement point. Examples of different categories into which each measurement point can be classified can be when the material is acceptable or when there are different flaws (missing layer, cracks, inhomogeneous layer, etc) in the material. Categorical classification of all measurement points provides the possibility of rapidly identifying incorrect areas within a material.

The choice of which categorical classification rule to use has previously been studied [14], usually selecting one of logistic regression or discriminant analysis. The discriminant analysis is really appropriate and valid only under the assumptions that the explanatory variables (i.e. the estimated model parameters) belong to a multivariate Gaussian distribution, and complete equality of the populations' covariance matrices [12]. The first criterion is rarely fulfilled in practice but can sometimes be enforced by some variable transformation. The assumption of equal covariance matrices is, however, typically not valid. Evaluating the performance of discriminant analysis is still relevant because of its simplicity compared to logistic regression. The main advantage with the logistic regression formulation is that the explanatory variables can have all kinds of forms (continuous, discrete, categorical). In an early study [14], the results showed that discriminant analysis had slightly higher efficiency when the assumptions were valid, but in all other situations it was outperformed by logistic regression. In this paper, we therefore compare the two methods. In the specific example in this paper, the rationale for the non-Gaussian distribution of the parameters is that some of them are reflection coefficients between layers of the material and these are, by definition, bounded as $|R| \leqslant 1$, and hence cannot be Gaussian.

The classification procedures, independently of which are used, differ depending on where they are applied, but mainly they differ due to the amount of available information about the entire set of measurement points. The classifiers have to be trained by using a representative set of measurement points from the different categories, for example by calibration measurements or by manually classifying a number of points. Measurement points from a new material are then selected into one of the predefined categories.

\subsubsection{Nominal logistic regression. When the dependent} variable is a set of categories which cannot be ordered in any meaningful way and consists of more than two categories, nominal logistic regression for more than two populations should be considered [12].

The basic idea with logistic regression is to estimate the probability $\widehat{p}_{j}$ of a measurement point belonging to each category $j$. Basically, for three categories, three probabilities are estimated and the category with the highest probability is chosen. The probabilities, $\widehat{p}_{1}, \widehat{p}_{2}, \ldots, \widehat{p}_{J}$, where $J$ is the number of categories, are estimated using the vector of estimated model parameters $\widehat{\boldsymbol{\theta}}$ from the multi-layered model and the estimated regression coefficients $\widehat{\boldsymbol{\beta}}_{1}, \ldots, \widehat{\boldsymbol{\beta}}_{J-1}$. When there are more than two categories, one of them is chosen as the comparison category, hence only $J-1$ regression coefficient vectors are required. The probabilities $p_{j}$ for $j=1, \ldots, J$ denote the probability for a measurement point to belong each category, that is, $p_{j}=\operatorname{Pr}\left(y_{i}=j\right)$, and are estimated as

$$
\begin{gathered}
\widehat{p}_{1}=\frac{1}{1+\sum_{j=1}^{J-1} \mathrm{e}^{\left[1, \widehat{\boldsymbol{\theta}}_{j}^{T}\right] \widehat{\boldsymbol{\beta}}_{j}}} \\
\widehat{p}_{i}=\frac{\mathrm{e}^{\left[1, \widehat{\boldsymbol{\theta}}_{i}^{T}\right] \widehat{\boldsymbol{\beta}}_{i}}}{1+\sum_{j=1}^{J-1} \mathrm{e}^{\left[1, \widehat{\boldsymbol{\theta}}_{j}^{T} \widehat{\boldsymbol{\beta}}_{j}\right.}}, \quad \text { for } \quad i=2, \ldots, J
\end{gathered}
$$

with the constraint that $p_{1}+p_{2}+\cdots+p_{J}=1$ for each measurement point. For $J$ categories, $J-1$ regression coefficient vectors need to be estimated, $\widehat{\boldsymbol{\beta}}_{1}, \ldots, \widehat{\boldsymbol{\beta}}_{J-1}$. The regression coefficient vectors $\widehat{\boldsymbol{\beta}}_{j}$ are the maximum likelihood estimates (MLEs) and are usually computed using an iteratively re-weighted least-squares (IRLS) approach [15], as

$$
\widehat{\boldsymbol{\beta}}_{j}=\widehat{\boldsymbol{\beta}}_{j, \text { old }}+\left(\widehat{\boldsymbol{\theta}}_{j}^{T} \mathbf{V}^{-1} \widehat{\boldsymbol{\theta}}_{j}^{T}\right)^{-1} \widehat{\boldsymbol{\theta}}_{j}\left(y_{j}-\widehat{p}_{j}\right),
$$

where $y_{j}$ is the known output category-dependent variable with fixed values, e.g. for three categories the values are $y_{1}=0$, $y_{2}=1$ and $y_{3}=2$. The weight matrix $\mathbf{V}$ is defined as [15]

$$
\mathbf{V}=\operatorname{Var}\left(\widehat{\boldsymbol{\theta}}_{j}^{T} \widehat{\boldsymbol{\beta}}_{j}\right)=\frac{1}{\widehat{p}_{j}\left(1-\widehat{p}_{j}\right)},
$$

which has the weighting elements as the diagonal entries. The initial guesses of the regression coefficient vectors $\widehat{\boldsymbol{\beta}}_{j}$ are usually all set to zero, resulting in equal probability for each category, but they could also be obtained by a suboptimal ordinary least-squares approach. The regression coefficient vectors $\widehat{\boldsymbol{\beta}}_{j}$ are then used to estimate the probabilities $\widehat{p}_{1}, \ldots$, $\widehat{p}_{J}$ in (4) and (5) for the estimated parameters $\widehat{\boldsymbol{\theta}}$ at new measurement points. Thresholding the results to the nearest predefined category, i.e. choosing the category with the highest probability, classifies each individual observation into one of the categories.

2.2.3. Discriminant analysis. The basic idea of discriminant analysis is to estimate the distances from an experiment to a number of predefined reference categories and classify the experiment to the closest category. The actual distance metric has a wide range of appearances [12], and in this study, where an experiment is an estimated parameter vector belonging to a measurement point, a quadratic distance metric is used. In a quadratic discriminant rule, the minimum squared distance from the estimated parameters at a new measurement point to each reference category is calculated. The function resulting in the minimum distance classifies the measurement point into that category. The quadratic distance is defined as [12]

$$
\widehat{d}_{j}=\frac{1}{2}\left(\widehat{\boldsymbol{\theta}}-\widehat{\boldsymbol{\mu}}_{j}\right)^{T} \widehat{\mathbf{C}}_{j}^{-1}\left(\widehat{\boldsymbol{\theta}}-\widehat{\boldsymbol{\mu}}_{j}\right)+\frac{1}{2} \log _{10}\left|\widehat{\mathbf{C}}_{j}^{-1}\right|+\log _{10}\left(r_{j}\right)
$$

for $j=1, \ldots, J$. In (8), $r_{j}$ is the a priori risk of misclassification and we have assumed that each reference 


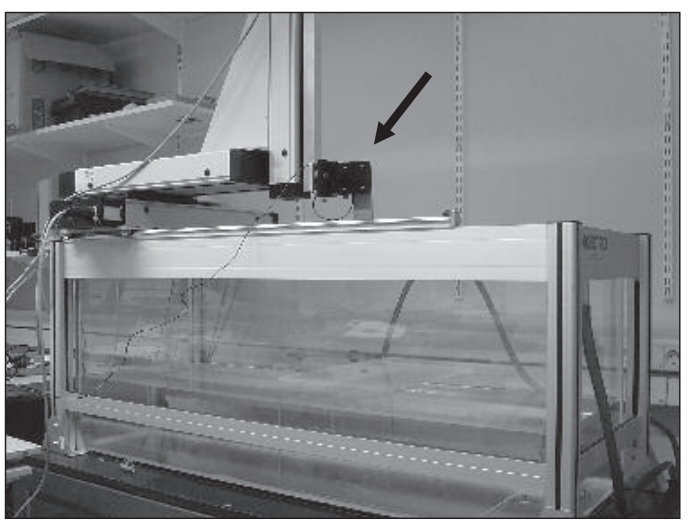

(a)

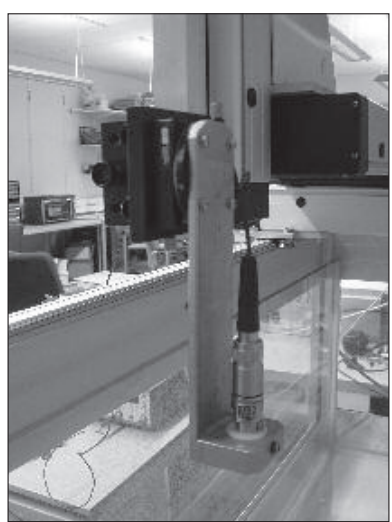

(b)

Figure 3. In $(a)$, the water tank and the steering axes are shown, and the arrow is pointing at the location where the transducer is mounted. In $(b)$, the mounting of the transducer is shown. During measurements the transducer was immersed in the water.

population has a multivariate normal distribution, i.e. $\boldsymbol{\theta}_{j} \sim$ $\mathcal{N}\left(\boldsymbol{\mu}_{j}, \mathbf{C}_{j}\right)$, where $\widehat{\boldsymbol{\theta}}$ is the vector of estimated parameters from a new point under investigation. In the classification, an equal risk of making misclassification is used, $r_{j}=0.5$.

2.2.4. Feature selection. The purpose of the feature selection step is to isolate the parameters having the most influence on the classification, that is, excluding the least significant parameters. This procedure strengthens the classification rule since only the most significant parameters are used, and might also simplify the procedure since fewer parameters are needed for the classification. A typical feature selection method is the backward elimination procedure which successively removes the least significant parameter [12]. The elimination procedure stops when all remaining parameters are statistically significant.

From the point of view of the physical modeling of the wave propagation in the material structure, all parameters are relevant and there is nothing to gain here by excluding some parameters. However, from a classification point of view, some of the parameters may be excluded. For example, if the classifier is trained to discriminate between different characteristics of the middle layer, model parameters related to the other layers may be non-informative. In other words, all parameters need to be estimated, but some may be excluded from the classification step.

\section{Experimental set-up}

The measurements were performed in a normal incidence pulse-echo configuration; see figure 2. All measurements were conducted with the ultrasound transducer, and the object was immersed in a 2001 water tank. The ultrasonic transducer was mounted on an arm connected to a steering motor with a spatial three-dimensional precision of approximately $12.5 \mu \mathrm{m}$ per step; see figure 3 . The step motor was then used to move the transducer in a plane over the material sample, in $1 \mathrm{~mm}$ steps in both the $x$ - and $y$-directions (see figure 4).

The temperature in the water was kept constant at $20.15^{\circ} \mathrm{C}$ to within $\pm 0.05^{\circ} \mathrm{C}$. For all the experiments performed in this

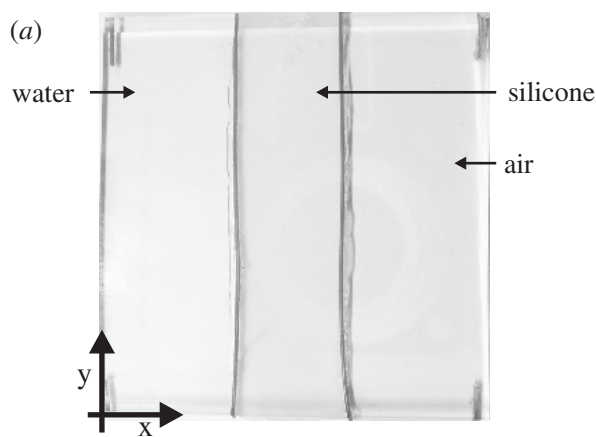

(b)

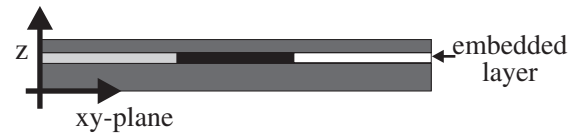

Figure 4. In (a), a photograph of the investigated material is shown, seen from above the material. The ultrasound transducer was used to scan the material sample in the $x$ - and $y$-directions. In $(b)$, an illustration of a cross-section of the material is shown. The thicknesses of the layers, in the $z$-direction, in this illustration are only descriptive and not the actual thicknesses. The gray area in the embedded middle layer corresponds to water, the black area is silicone and the white area is air.

work, a $5 \mathrm{MHz}$ non-focused piezoelectric transducer (V3456) from Panametrics (Waltham, MA, USA) with an element size of $13 \mathrm{~mm}$ was used. To excite and receive acoustic pulses, a Panametrics Pulser/Receiver Model 5073PR operating in pulse-echo mode was used. The reflected ultrasonic echoes were sampled using a Gage CompuScope CS12400 (Lachine, QC, Canada), with a sampling frequency of $200 \mathrm{MHz}$ and a 12 bit resolution.

The examined object consisted of two Pyrex glass layers bonded together by a thin layer. The thickness of the top glass layer was $1.8 \pm 0.05 \mathrm{~mm}$ and the thickness of the bottom glass layer was $3.8 \pm 0.05 \mathrm{~mm}$. The thickness of the embedded middle layer was fixed in distance at $0.5 \pm 0.05 \mathrm{~mm}$. A mixture of construction silicone was used as a bonding material. Flaws, present as air and water gaps, contribute to inhomogeneity in the bonding layer. Figure 4 shows a photograph of the sample used in the measurements and an illustration of a cross-section 


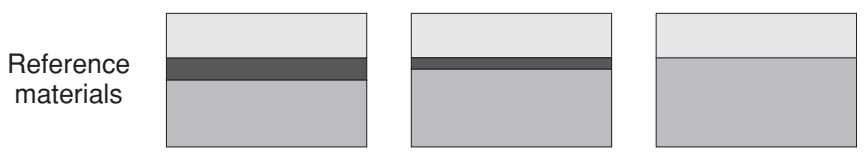

Test

material

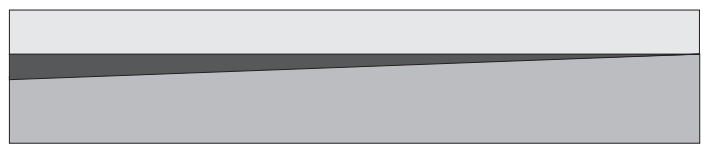

Figure 5. An illustration of the cross-sections of the three different simulated training materials with varying thickness of the embedded middle layer: full thickness, half thickness and zero. Below is a schematic view of the simulated test material with slowly decreasing thickness of the middle layer.

of the material. The material is created by gluing the two Pyrex glass layers together using silicone only in the middle part of the material, at a fixed distance controlled by two bars. The water flaw area is then created by leaving the edges open, so when the object is immersed in the tank, the water will be present in that area. The air flaw area is created by sealing the edges with silicone before the object is immersed in the tank, hence air is present in that area.

\section{Simulation results}

For the simulations, samples were created with similar structures to those used in the measurements. The materials were defined by the thickness, the speed of sound, the density and the attenuation coefficient in every layer. Random variations were incorporated in all of these quantities simulating materials with more realistic properties. The purpose of the simulations was to evaluate the performance of the classifier for a slowly decreasing thickness of the middle layer. Basically, the task was to classify a new material into one of the three categories: full thickness of the layer (category zero, $c_{0}$ ), half thickness of the layer (category one, $c_{1}$ ) and missing layer (category two, $c_{2}$ ).

A short ultrasound pulse was transmitted through the material, producing an output consisting of several overlapping pulses (with some measurement noise added). The simulated output signals from each measurement point were then used when estimating the parameter vectors corresponding to each location on the sample. The classifier was trained using the three different reference materials, and then the measurement points of the test material were classified into one of the categories defined by the reference materials. Figure 5 shows a principal illustration of the simulated material samples. The test sample was created with a slowly decreasing thickness of the middle layer, from $110 \mu \mathrm{m}$ to zero, and an equally slowly increasing thickness of the bottom layer from $0.5 \mathrm{~mm}$ to $0.61 \mathrm{~mm}$. The thickness of the top layer was kept constant at $1 \mathrm{~mm}$. The simulations used a narrow sound field, and hence the model assumption of parallel layers is still reasonable.

In this simulation set-up, the only parameters that varied were the times-of-flight in the middle and bottom layers, $\widehat{\tau}_{2}$ and $\widehat{\tau}_{1}$. The feature selection procedure revealed that the parameter $\widehat{\tau}_{2}$ should be enough for classification, which

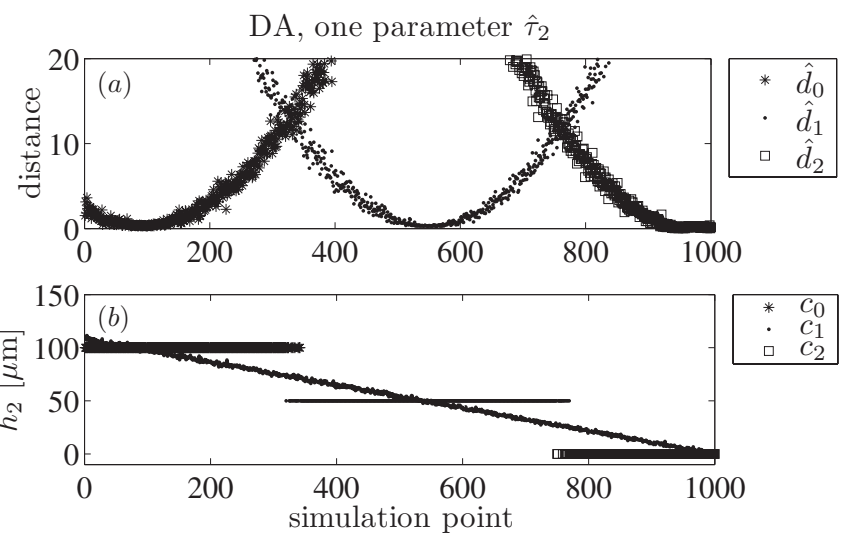

Figure 6. Classification results from the measurements using discriminant analysis. In $(a)$ the quadratic distances from the simulation points to the three categories are shown. In $(b)$ the decreasing thickness, $h_{2}$, of the middle layer is shown together with a visualization of which category, $c_{0}, c_{1}$ or $c_{2}$, the points are classified into.
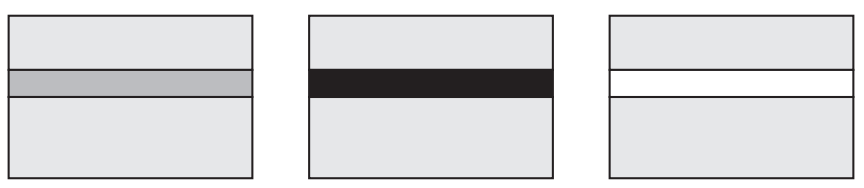

Figure 7. An illustration of the cross-sections of the three training samples with the embedded middle layer being either (from left to right): water, construction silicone or air.

makes sense, since the two parameters do not really contribute with independent information when the total thickness of the sample is constant. Since this parameter, in these simulations, belongs to a multivariate Gaussian distribution and has similar covariance matrices (between each population), logistic regression and discriminant analysis should give similar results and due to its simplicity we focus on discriminant analysis. In figure 6 , the classification results are shown for this simulation, and in $6(a)$ the estimated distance to each category is shown. In figure $6(b)$ the decreasing thickness, $h_{2}$, of the middle layer is shown together with a visualization of which category each point was classified into. The points are classified into a new category about halfway between the reference categories, that is at thicknesses $\sim 75 \mu \mathrm{m}$ and $\sim 25 \mu \mathrm{m}$. Adding more reference categories in between the present ones would enhance precision but would also increase the number of uncertain estimates.

\section{Measurement results}

\subsection{Training materials and data arrangement}

Figure 4 shows a photograph of the sample used in order to evaluate the proposed classification methods. The sample consists of three layers with fixed dimensions (see section 3) with the middle layer made up of three different materials: water, silicone and air. To train the classifiers, three separate samples were constructed, all having the same dimensions as the test sample (see figure 7). 
Table 1. The explanatory variables for the reference/training materials.

\begin{tabular}{lll}
\hline Category & $\begin{array}{l}\text { Measurement } \\
\text { point }\end{array}$ & $\begin{array}{l}\text { Explanatory } \\
\text { variables }\end{array}$ \\
\hline 0 & 1 & $\widehat{\boldsymbol{\theta}}_{1}^{T}$ \\
$\vdots$ & $\vdots$ & $\vdots$ \\
0 & $N$ & $\widehat{\boldsymbol{\theta}}_{N}^{T}$ \\
1 & $N+1$ & $\widehat{\boldsymbol{\theta}}_{N+1}^{T}$ \\
$\vdots$ & $\vdots$ & $\vdots$ \\
1 & $2 N$ & $\widehat{\boldsymbol{\theta}}_{2 N}^{T}$ \\
2 & $2 N+1$ & $\widehat{\boldsymbol{\theta}}_{2 N+1}^{T}$ \\
$\vdots$ & $\vdots$ & $\vdots$ \\
2 & $3 N$ & $\widehat{\boldsymbol{\theta}}_{3 N}^{T}$ \\
\hline
\end{tabular}

The training samples were scanned in a $10 \times 10 \mathrm{~mm}^{2}$ area with $1 \mathrm{~mm}$ step, and the model parameters were then estimated for each measurement point, resulting in $N=100$ experiments for each object. The parameters were used as the explanatory variables and are arranged as shown in table 1 , where the column vector $\widehat{\boldsymbol{\theta}}$ contains the estimated model parameters. The estimated parameter vectors for each training material were assigned categories 0,1 and 2 for water, silicone and air, respectively.

The test object in figure 4 was then scanned in a $50 \times$ $50 \mathrm{~mm}^{2}$ area with a step size of $1 \mathrm{~mm}$, resulting in 2500 measurements. For each of these, the 10 model parameters (see equation (2), section 2.1), $\boldsymbol{\theta}$, were estimated and stored in a $2500 \times 10$ matrix, $\mathbf{X}$.

\subsection{Data analysis}

The first step of the analysis is to check if the estimated model parameters $\widehat{\boldsymbol{\theta}}$ can be assumed to belong to a multivariate Gaussian distribution, since this will affect the performance of classification methods. The normality assumption is tested for all parameters, but here we only display a representative selection. Figures $8(a)$ and $(b)$ show scatter plots of the parameter $\widehat{\tau}_{2}$ against $\widehat{\tau}_{3}$ and $\widehat{R}_{32}$, i.e. the time-of-flight through the top and the middle layers, and the reflection coefficient between the top and the middle layers. From figure $8(a)$ it is evident that $\widehat{\tau}_{2}$ and $\widehat{\tau}_{3}$ do not follow a $2 \mathrm{D}$ normal distribution. Figure $8(b)$ is more difficult to interpret, although it seems unlikely that $\widehat{\tau}_{2}$ and $\widehat{R}_{32}$ belong to a $2 \mathrm{D}$ normal distribution. Figures $8(c)$ and $(d)$ show normal probability plots of $\widehat{\tau}_{2}$ and $\widehat{\tau}_{3}$, and we see that while $\widehat{\tau}_{2}$ seems to be normally distributed, $\widehat{\tau}_{3}$ does not. The same procedure can be followed for the other parameters. However, already now we can conclude that the logistic regression is a better choice than discriminant analysis. Nevertheless, both will be evaluated.

\subsection{Nominal logistic regression}

First, the model parameters estimated from the measurement on the training samples are used to estimate the regression coefficients $\widehat{\boldsymbol{\beta}}_{j}$ in equation (6). The regression coefficients
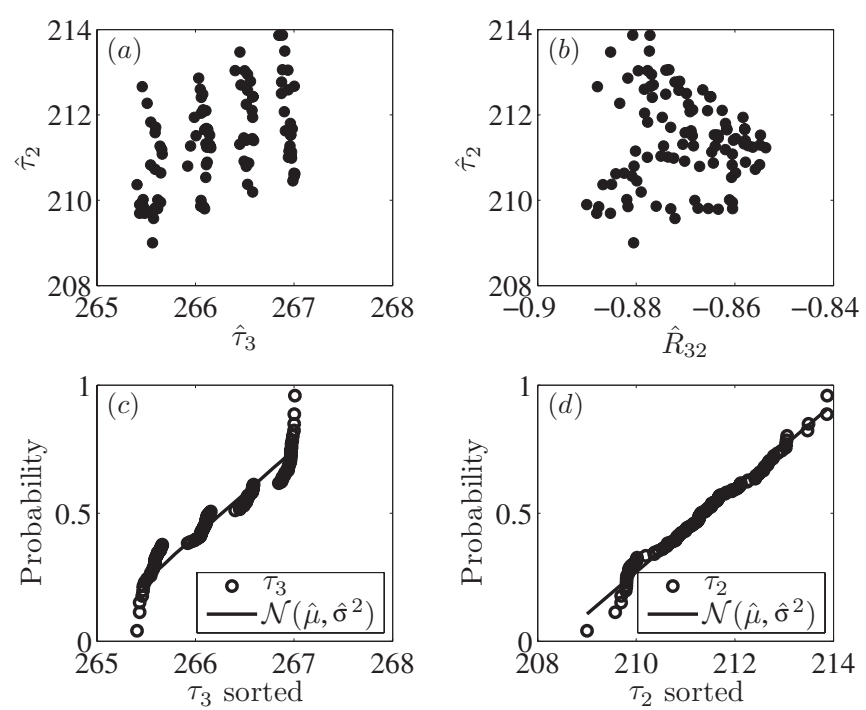

Figure 8. In (a) the scatter plot with parameters $\widehat{\tau}_{2}$ and $\widehat{\tau}_{3}$ reveals the parameters not to be multivariate normal distributed which cannot be said in $(b)$. In $(c)$ and $(d)$ normal probability plots of $\widehat{\tau}_{3}$ and $\widehat{\tau}_{2}$ reveal the first not to be normal distributed.

Table 2. Properties of the classifier.

\begin{tabular}{lrll}
\hline Predictor & \multicolumn{1}{c}{$\widehat{\beta}$} & $\widehat{\sigma}_{\beta}$ & $P$-value \\
\hline Logit 1: $(1 / 0)$ & & & \\
Constant & -354.2 & 28420 & 0.990 \\
$\widehat{R}_{32}$ & 365.6 & 25730 & 0.989 \\
$\widehat{\tau}_{2}$ & 3.321 & 120.0 & 0.978 \\
Logit 2: $(2 / 0)$ & & & \\
Constant & -42.24 & 9.529 & 0.000 \\
$\widehat{R}_{32}$ & -27.20 & 12.79 & 0.033 \\
$\widehat{\tau}_{2}$ & 0.095 & 0.04552 & 0.037 \\
\hline
\end{tabular}

and the experiment matrix $\mathbf{X}$ are then used to estimate the probabilities, $\widehat{p}_{1}, \ldots, \widehat{p}_{J}$ in (4) and (5), of each measurement point belonging to the different categories. The experiment matrix $\mathbf{X}$ contains vectors with the estimated model parameters for every location on the material.

In table 2, the classification results are shown. Since there are three categories we obtain two regression coefficient vectors, $\widehat{\boldsymbol{\beta}}_{1}$ and $\widehat{\boldsymbol{\beta}}_{2}$. The first vector is estimated using the estimated parameters from category 1 and the reference category 0 (Logit 1 , in table 2 ) and the second using the estimated parameters from category 2 and the reference category 0 (Logit 2 , in table 2 ). The regression coefficients $\widehat{\boldsymbol{\beta}}$ and the corresponding standard deviation $\widehat{\sigma}_{\beta}$ are presented. Also given in the table are the $P$-values for each parameter, for which a low value $(P<0.05)$ indicates that the coefficient cannot be excluded. A low $P$-value indicates that we can reject the null hypothesis, which is that the parameter is not significant. All coefficients are significant for the second coefficient vector $\widehat{\boldsymbol{\beta}}_{2}$, see $\widehat{\sigma}_{\beta_{2}}$ and $P$, hence they cannot be excluded.

The backward elimination feature selection suggested that only the parameters $\widehat{R}_{32}$ and $\widehat{\tau}_{2}$ were significant (see table 2 ), 

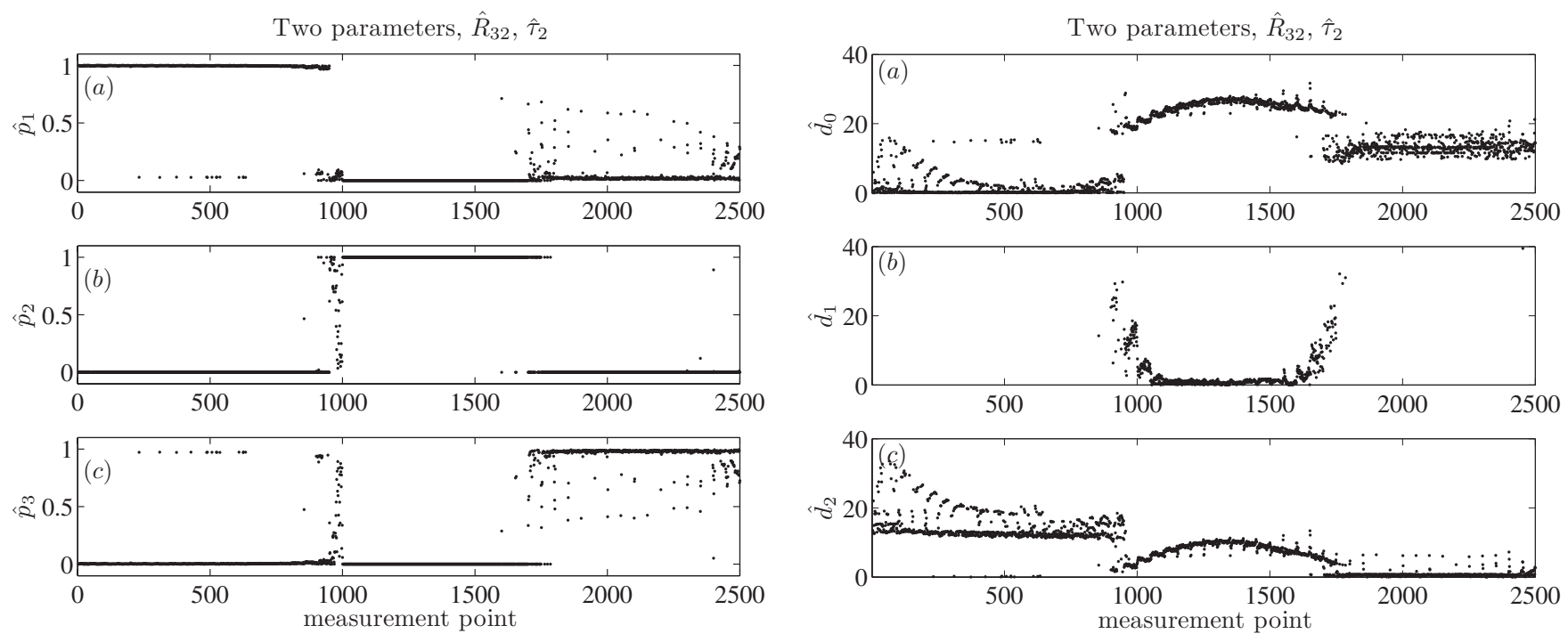

Figure 9. The result from the classification of all measurement points using logistic regression. The probability of each category is plotted for each measurement point on the object.

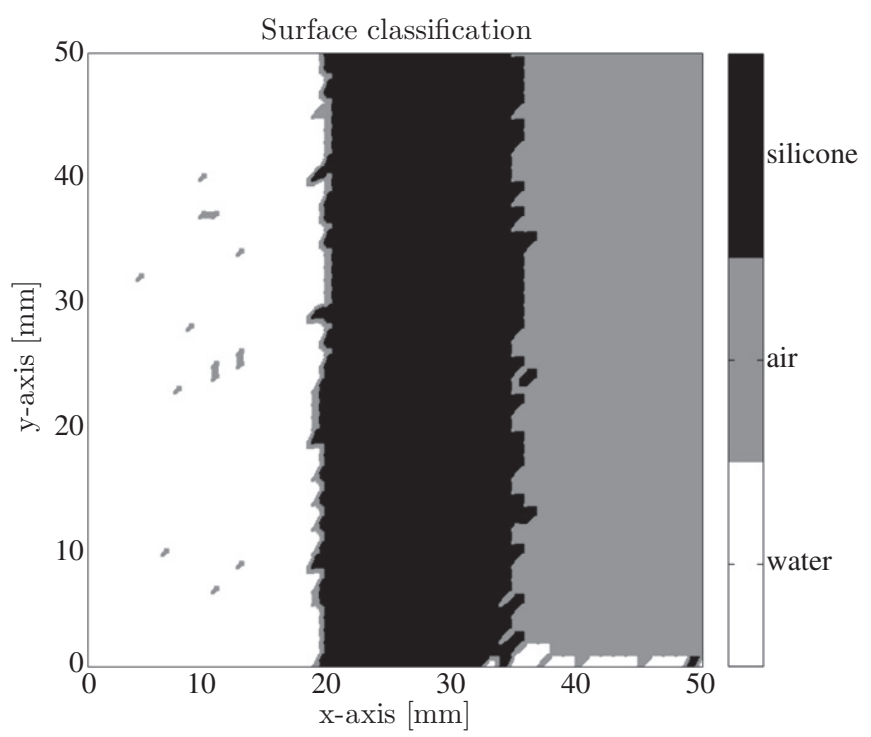

Figure 10. The result from the classification of the measurements using logistic regression. The highest probability of each category was assigned a different gray level ( silicone $=$ black, water $=$ white and air $=$ gray).

which is reasonable since these are the parameters related to the middle layer. The classification results are shown in figure 9.

Figure 10 shows the classification result as a surface plot. Comparing this with the real object in figure 4 we see that the classifier assigns most measurement points to the correct category. However, since the exact properties of the test object are unknown, it is not possible to calculate any percentage of correctly classified points.

\subsection{Discriminant analysis}

Again, all measurement points on the material in figure 4 are to be classified. The reference materials are used to estimate

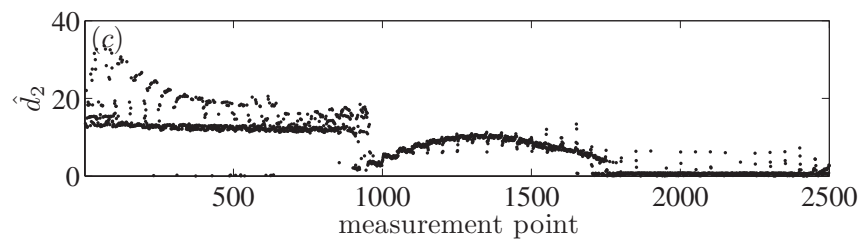

Figure 11. The result from the classification of all measurement points using discriminant analysis. The distance of each category is plotted for each measurement point on the object. The distances over 40 are not visible in the figures and not required when choosing the shortest distance.

the means $\widehat{\boldsymbol{\mu}}_{j}$ and the covariance matrices $\widehat{\mathbf{C}}_{j}$. The experiment matrix $\mathbf{X}$ together with the means and covariance matrices is then used to estimate the distances between each measurement point and each of the categories, $\widehat{d}_{0}, \widehat{d}_{1}$ and $\widehat{d}_{2}$ in (8). The classification rule is to choose the category with the shortest quadratic distance.

Backward elimination is used for feature selection and the best decision rule was found to be using two parameters, $\widehat{R}_{32}$ and $\widehat{\tau}_{2}$. These are the same parameters as in the nominal logistic regression classifier. The classification results are shown in figure 11. Using the same interpretation as in section 5.3, the classification results can be viewed as a surface plot. Comparing this surface plot, figure 12, with the real object in figure 4 reveals a significant match. However, when we compare figures 10 and 12 we see that the logistic regression performs slightly better. The reason for this, as was also explained in the theory section, is that discriminant analysis requires the parameters to be normally distributed. Since we know this is not the case, logistic regression should perform better. However, depending on the requirements of the classifier, the reduced computational complexity might still motivate the use of discriminant analysis.

\section{Discussion}

The classification results from the two classifiers were approximately equal after feature selection had been incorporated, as can be seen in sections 5.3 and 5.4. However, if the classification is done without incorporating the feature selection procedure, logistic regression outperforms discriminant analysis. The main reason is the fact that 


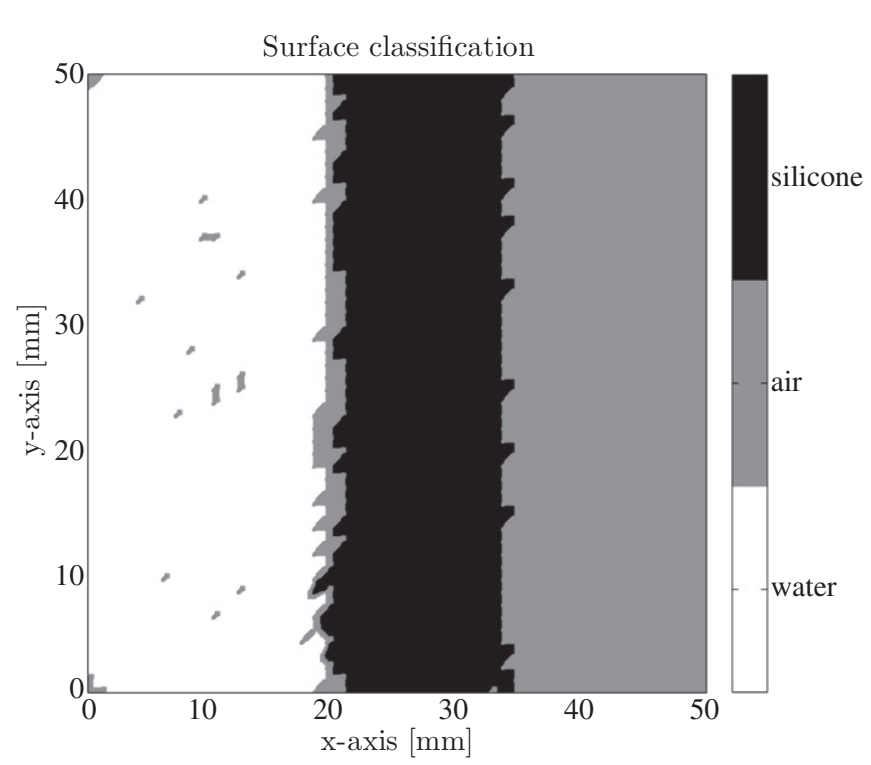

Figure 12. The result from the classification of the measurements using discriminant analysis. The shortest distance to each category is assigned a discrete value and plotted at each measurement point on the object. The water region is assigned 0 (white), silicone 1 (black) and air 0.5 (gray).

in feature selection, non-multivariate normal distributed parameters are excluded, bringing the performance of the two different classifiers together. The two parameters remaining after feature selection are both close to normally distributed, see figures $8(b)$ and $(d)$. Discriminant analysis is simpler, only calculating distances without requiring iterative parameter estimations as in logistic regression, indicating that choosing discriminant analysis above logistic regression might be reasonable, despite the possible violation of multivariate normally distributed parameters. However, with the computer power available today this will not be an issue for most practical applications.

The parameters remaining after feature selection in this study are highly connected to the flaw areas. If errors were located in other locations inside the material or if additional unknown materials or flaws were present, different parameters would be revealed as significant by the feature selection procedure. The importance of training the classifier with representative reference materials for all possible material types must therefore be emphasized. However, there is a tradeoff between the number of categories and the possibility of classifying into the correct category. For localized flaw areas one can argue that studying some of the parameters directly will be enough, but for more complicated material and flaw structures an automated classifier is beneficial.

It should also be noted that the classifiers presented here merely demonstrate the principle, i.e. that different types of materials or flaws can be discriminated using only the parameters of the wave propagation model. The classifiers could, of course, also be trained to discriminate between other features of the material samples. If the method presented here is to be used as a tool for online process control, it is likely that the classification rules are modified as the user learns more about the process. In combination with traditional destructive tests, more and more features can be incorporated into an automated classification procedure such as that proposed here.

\section{Conclusions}

In this paper, we have shown how the use of a parametric model of the propagation of pulse-echo ultrasound through thin layers of multi-layered materials enables automatic classification. Two classification methods were evaluated: discriminant analysis and logistic regression. The classifiers were first trained using representative samples of the material. After that the results of applying the classifiers on a test object were evaluated. The results show that both classifiers perform well, although the performance of the logistic regression was slightly better than that based on discriminant analysis. The reasons for this were also discussed in the paper.

The possibility of training the classifier based only on the parameters of the wave propagation is especially valuable in an online process control application since the data reduction is substantial, and once the calibration is done the computations are fast. The results also show that the model parameters carry enough information about variations in the material properties to be used for flaw/material classification.

\section{References}

[1] Papadakis E P 1988 Online statistical process control with NDE and computers Proc. IEEE Int. Ultrasonics Symp. (Chicago, IL: IEEE) pp 523-7

[2] Heyman J S, Winfree W P, Parker F R, Heath D M and Welch C S 1989 Quantitative NDE applied to composites and metals Proc. Symp. Nondestructive Monitoring of Materials Properties Symposium (Boston, MA 28-30 November 1988) 211-20

[3] Mehrabian R and Wadley N G 1985 Needs for process control in advanced processing of materials J. Met. 37 51-8

[4] Freemantle R, Challis R and White J 1994 A Z-transform technique for thin-layer reverberation cancellation applied to ultrasonic NDT of adhered structures IEE Colloquium on Advanced Techniques for Collection and Interpretation of NDT Data vol 102 pp 7/1-4

[5] Wang L, Xie B and Rokhlin S 2002 Determination of embedded layer properties using adaptive time-frequency domain analysis J. Acoust. Soc. Am. 111 2644-53

[6] Martin C, Meister J J, Arditi M and Farine P A 1992 A novel homomorphic processing of ultrasonic echoes for layer thickness measurement IEEE Trans. Signal Process. 40 1819-25

[7] Kinra V and Iyer V 1995 Ultrasonic measurement of the thickness, phase velocity, density or attenuation of a thin-viscoelastic plate: part I. The forward problem Ultrasonics 33 95-109

[8] Kinra V and Iyer V 1995 Ultrasonic measurement of the thickness, phase velocity, density or attenuation of a thin-viscoelastic plate: part II. The inverse problem Ultrasonics 33 111-22

[9] Kundu T 1992 Complete acoustic microscopical analysis of multilayered specimens J. Appl. Mech. 59 54-60

[10] Rokhlin S and Huang W 1992 Ultrasonic wave interaction with a thin anisotropic layer between two anisotropic solids: exact and asymptotic-boundary-condition methods J. Acoust. Soc. Am. 92 1729-42 
[11] Hägglund F, Martinsson J and Carlson J E 2009 Model-based estimation of thin multi-layered media using ultrasonic measurements IEEE Trans. Ultrason. Ferroelectr. Freq. Control 56 1689-702

[12] Johnsson D E 1998 Applied Multivariate Methods for Data Analysis (Belmont, CA: Duxbury Press)
[13] Kinsler L E, Frey A R, Coppens A B and Sanders J V 1982 Fundamentals of Acoustics 3rd edn (New York: Wiley)

[14] Press S J and Wilson S 1978 Choosing between logistic regression and discriminant analysis J. Am. Stat. Assoc. 73 699-705

[15] Hosmer D W and Lemeshow S 1989 Applied Logistic Regression (New York: Wiley) 